\title{
Pregnancy and Perinatal Outcomes Associated with Acinetobacter baumannii Infection
}

\author{
Mai He, MD, PhD ${ }^{1}$ Stefan Kostadinov, MD ${ }^{1}$ Fusun Gundogan, MD ${ }^{1}$ \\ Judith Struminsky, MT(ASCP), MBA ${ }^{1}$ Halit Pinar, MD ${ }^{1}$ \\ C. James Sung, $\mathrm{MD}^{1}$ \\ ${ }^{1}$ Department of Pathology and Laboratory Medicine, Women and \\ Infants Hospital of Rhode Island, The Warren Alpert Medical School of \\ Brown University, Providence, Rhode Island \\ Address for correspondence Mai He, MD, PhD, Department of \\ Pathology and Laboratory Medicine, Women and Infants Hospital of \\ Rhode Island, The Warren Alpert Medical School of Brown University, \\ 101 Dudley Street, Providence, RI 02905 \\ Am J Perinatol Rep 2013;3:51-56. \\ (e-mail: hemaipath@yahoo.com).
}

\begin{abstract}
Keywords

- autopsy

- stillbirth

- placenta

- pregnancy

- bacterial culture

Objective To determine perinatal and pregnancy outcomes of Acinetobacter baumannii infection using clinicopathologic material from pregnant women, neonates, and perinatal postmortem examinations with positive cultures.

Study Design This is a retrospective record review with placental and postmortem examination.

Results During a 5-year period, 40 positive cultures were found. Three pregnancies with positive cultures close in the peripartum period were all associated with adverse outcomes including spontaneous abortion, preterm labor, and one full-term birth with histological chorioamnionitis. Two positive cultures were found in preterm neonates in the neonatal intensive care unit. Two of three cases of perinatal death grew pure cultures from blood and/or fetal tissue with placental or fetal examination demonstrating evidence of infection/inflammation with fetal inflammatory response.

Conclusion This is the first case series report of $A$. baumannii-positive cultures in maternal, fetal, and neonatal specimen, with histopathologic evidence of infection. The results suggest a significant role of $A$. baumannii infection in adverse pregnancy and perinatal outcomes.
\end{abstract}

Acinetobacter species are a group of bacterial microorganisms that have emerged as significant nosocomial pathogens, with Acinetobacter baumannii being the most frequently isolated species. Acinetobacter infections have attracted increasing attention during recent years because they are resistant to most antibiotics and can be found in both hospitalized patients and the community. The spectrum of clinical manifestations is broad. In general, A. baumannii mainly infects patients with impaired host defense, such as those who are in the intensive care units. ${ }^{1}$ Although there are conflicting results regarding clinical outcomes from different clinical studies, it appears that $A$. baumannii infection is associated with increased mortality. 2,3
Patients in neonatal intensive care units (NICU) usually have medical conditions that are associated with impaired host defense or immune response, such as preterm and premature birth. These conditions make them vulnerable for $A$. baumannii infection. There are a few reports of outbreaks of $A$. baumannii in the NICU. ${ }^{4,5}$ Although death of infected neonates was reported in some of the studies, so far there is no report in the literature of postmortem examination with perinatal death associated with cultures positive for A. baumannii. The effect of A. baumannii infection on pregnancy was rarely studied; so far there is only one case report regarding $A$. baumannii infection related to pregnancy. 6 received

July 25,2012

accepted

July 27, 2012

published online

February 7, 2013
Copyright $\odot 2013$ by Thieme Medical Publishers, Inc., 333 Seventh Avenue, New York, NY 10001, USA. Tel: +1(212) 584-4662.
DOI http://dx.doi.org/ 10.1055/s-0033-1334460. ISSN 2157-6998. 
Our objective was to determine perinatal and pregnancy outcomes of $A$. baumannii infection using clinicopathologic material from pregnant women, neonates, and perinatal postmortem examinations (autopsy) with positive bacterial cultures.

\section{Materials and Methods}

\section{Design}

This was a retrospective record review from the beginning of 2007 to the end of 2011 ( 5 full years).

\section{Hospital Setting}

The hospital is a regional tertiary hospital specializing in health care of women and newborns.

\section{Methods}

With approval from the Institutional Review Board, a keyword search for "Acinetobacter baumannii" was performed in the health information system for any bacterial cultures positive for A. baumannii during the study period. Cases identified were further classified by source of culture into three groups: adult, newborn units, and autopsy (postmortem examination). Primary focus of study was cultures from perinatal death, neonates, and pregnancy-related cases.

Positive bacterial cultures from adults included both outpatients and inpatients. Clinical history was extracted to determine if the culture was obtained during pregnancy or immediately postpartum. For pregnancy-related cultures, gestational age (weeks) when the cultures were sampled and delivery took place were recorded to judge whether the positive culture could be related to the outcome. If the culture was obtained around the time of labor, or within 4 weeks before labor, it was judged as "most likely relevant," otherwise it was judged as "probably not."

Newborn units include the NICU and regular newborn nursery. Postmortem examination of perinatal death included both stillbirth and neonatal death in the NICU. For any postmortem (autopsy) cases identified, available maternal bacterial cultures and related clinical histories and postmortem findings, including placental examination results, were obtained and summarized.

\section{Histological Analysis and Definition of Terminology} Postmortem examination was performed following established protocol. ${ }^{7,8}$ Briefly, it consisted of gross and microscopic examination of fetus or infants, placenta, and auxiliary tests such as bacterial cultures and cytogenetic study.

\section{Amniotic Fluid Infection Syndrome}

Evidence of infection/inflammation of the extraplacental membranes and chorionic plate of the placenta, inflammatory cells in lung parenchyma or gastrointestinal (GI) tract lumen, and/or positive postmortem cultures were examined.

\section{Histological Evidence of Fetal Inflammatory Response} Vasculitis of fetal blood vessel at either umbilical cord or chorionic plate was recorded.

\section{Funisitis}

Inflammation of Wharton's jelly of umbilical cord was recorded.

\section{Results}

\section{Clinical Features of Positive Bacterial Cultures for}

\section{A. baumannii}

Overall bacterial infection from A. baumannii was rare. During the study period (January 1, 2007, to December 31, 2011), there were 40 bacterial cultures in total positive for A. baumannii. These included 33 cultures from adults, three cultures from newborn units, and four cultures from postmortem examinations, as summarized in - Table 1. Classified by the type of specimen, voided urine was the most common sample (16, 40\%), followed by wound (13, 32.5\%). All cases were isolated; there were no outbreaks.

\section{Clinical Outcomes of Positive Cultures Related to Pregnancy}

Among the 33 positive cultures from adults, 7 specimens from 6 patients $(7 / 33,21.2 \%)$ were related to pregnancy, as summarized in - Table 2. Maternal age ranged from 22 to 34 years old. There were 5 voided urine samples $(5 / 7,71.4 \%), 1$ catheter tip, and 1 breast milk sample (1/7, 14.3\%). Five of the six patients had placenta examination reports. Patient 5 had no pathological examination and was clinically diagnosed as spontaneous abortion (SAB). Four pregnancies ended up with term labor, one with preterm labor, and one with SAB.

Three cases had 4 positive cultures around the time of labor or within 4 weeks before labor (case 6 had two cultures). Case 1 had urine culture growing pure A. baumannii around the time of labor, and placental examination revealed acute chorioamnionitis at fetal membrane and chorionic plate. Case 5 had urine culture growing Escherichia coli and A. baumannii during the clinical visit and was clinically diagnosed as SAB. Case 6 had history of chronic hypertension and intrauterine growth restriction during that pregnancy. The patient had two urine cultures growing pure A. baumannii at 24 and 25 gestation weeks. She was treated with sulfamethoxazole and trimethoprim and completed the course. The patient went

Table 1 Clinical Features of Bacterial Cultures Positive for Acinetobacter Baumannii

\begin{tabular}{|l|l|l|l|}
\hline Source & $\begin{array}{l}\text { Voided urine } \\
(n=16)\end{array}$ & $\begin{array}{l}\text { Wound } \\
(n=13)\end{array}$ & $\begin{array}{l}\text { Others } \\
(n=11)\end{array}$ \\
\hline Adults $(n=33)$ & & & \\
\hline Inpatient $(n=12)$ & 5 & 4 & $3^{\mathrm{a}}$ \\
\hline Outpatient $(n=21)$ & 11 & 8 & $2^{\mathrm{b}}$ \\
\hline Newborns $(n=3)$ & 0 & 1 & $2^{\mathrm{c}}$ \\
\hline Autopsy $(n=4)$ & 0 & 0 & 4 \\
\hline
\end{tabular}

${ }^{a}$ Breast milk, lung aspiration and catheter tip.

bThroat, sputum.

${ }^{\mathrm{c}}$ Tracheal aspiration, blood from central line. 
Table 2 Clinical Features with Cultures Positive for Acinetobacter Baumannii During Pregnancy

\begin{tabular}{|c|c|c|c|c|c|c|c|}
\hline Case & $\begin{array}{l}\text { Maternal } \\
\text { age }(y)\end{array}$ & Type of culture & $\begin{array}{l}\text { Gestational age } \\
\text { for positive } \\
\text { culture (wk) }\end{array}$ & $\begin{array}{l}\text { Gestational } \\
\text { age at } \\
\text { birth (wk) }\end{array}$ & Outcome & Placental findings & Relevant? \\
\hline 1 & 22 & Voided urine & 40 & 40 & Term birth & $\begin{array}{l}\text { SGA, acute } \\
\text { chorioamnionitis }\end{array}$ & $\begin{array}{l}\text { Most likely } \\
\text { yes }\end{array}$ \\
\hline 2 & 34 & Voided urine & 32 & 39 & Term birth & $\begin{array}{l}\text { No significant } \\
\text { findings }\end{array}$ & Probably not \\
\hline 3 & 29 & Catheter tip & 10 & 37 & Term birth & $\begin{array}{l}\text { No significant } \\
\text { findings }\end{array}$ & Probably not \\
\hline 4 & 24 & Breast milk & Postpartum & 40 & Term birth & $\begin{array}{l}\text { SGA, acute vasculitis } \\
\text { of umbilical cord }\end{array}$ & Probably not \\
\hline 5 & 27 & Voided urine & Early pregnancy & $\begin{array}{l}\text { Not } \\
\text { applicable }\end{array}$ & $\mathrm{SAB}$ & Not applicable & $\begin{array}{l}\text { Most likely } \\
\text { yes }\end{array}$ \\
\hline 6 & 30 & Voided urine & 24,25 & 28 & Preterm labor & $\begin{array}{l}\text { Decidual vasculopathy, } \\
\text { fetal thrombotic } \\
\text { vasculopathy }\end{array}$ & $\begin{array}{l}\text { Most likely } \\
\text { yes }\end{array}$ \\
\hline
\end{tabular}

Abbreviation: SGA, small for gestational age.

into labor at 28 weeks' gestation. Placental examination revealed no evidence of chorioamnionitis.

In summary, adverse pregnancy outcomes were seen in all three pregnancies with positive A. baumannii cultures around the time of labor.

\section{Clinical Features of Positive Cultures from Newborn Units}

Three positive cultures for A. baumannii were found from newborn units, including two cases from the NICU (cases A and $B$ ) and one from the newborn nursery (case C).

NICU case A was a girl born at 24 weeks with birth weight of $780 \mathrm{~g}$. There was no evidence of infection or inflammation in placenta. On day of life (DOL) 31, she developed temperature instability. Blood culture from the central line was positive for Staphylococcus capitis and respiratory culture was positive for A. baumannii. She was initially started on oxacillin and gentamicin and completed a 7-day course. The central line was removed and there was no more positive culture. The patient was discharged on DOL 103.

NICU case B was a baby girl born at 31 weeks' gestational age with birth weight of $1,500 \mathrm{~g}$. She was admitted to the NICU with prematurity, respiratory distress, and congenital cytomegalovirus infection. On DOL 20, blood from the central line was positive for $A$. baumannii. The infant was started on ganciclovir, ampicillin, and gentamicin. Antibiotics were discontinued after 2 days of no growth of blood cultures. She was discharged home on DOL 50.

One positive culture was found from a scalp abscess from a full-term newborn (case $C$ ) in the newborn nursery. The hospital course was otherwise unremarkable.

\section{Brief Clinical Histories and Postmortem Findings of Autopsies}

Of 509 cases of perinatal autopsy during the study period, there were four A. baumannii-positive cultures from three postmortem examinations (3/509, 0.6\%, postmortem cases 1 to 3 ). Clinical histories and postmortem findings of three autopsies are summarized in - Table 3 . The birth weight in all three cases was below $1,000 \mathrm{~g}$.

The postmortem case 1 was a female neonate born at 23 weeks' gestation to a 25 -year-old gravida 2, para 1 without significant past medical history. Birth weight was $590 \mathrm{~g}$ (50th to 90th percentile, Hadlock Fetal Growth Curves). Placental examination revealed severe acute chorioamnionitis with three-vessel acute vasculitis and funisitis (inflammation of Wharton's jelly) of the umbilical cord and acute vasculitis of chorionic fetal blood vessels. The newborn developed complications of prematurity and lived 289 days. Her hospital course included bowel perforation at DOL 9 and multiple episodes of sepsis. Postmortem examination revealed multiorgan failure with cultures growing multiple organisms. A. baumannii grew from peritoneal effusion with Enterococcus species and Neisseria sicca.

The postmortem case 2 was a female newborn born at 21 weeks' gestation to a 27 -year-old gravida 2, para 0 mother with history of preterm premature rupture of membranes (PPROM) at 15 to 20 weeks' gestation. Birth weight was $375 \mathrm{~g}$ (10th to 50th percentile, Hadlock Fetal Growth Curves). She lived less than 10 minutes. The mother presented with cramping since the early morning but denied fever or chills. Postmortem examination revealed evidence suggestive of amniotic fluid infection syndrome, including placenta with severe acute chorioamnionitis and acute vasculitis of chorionic fetal vessels (-Fig. 1A and 1B) and a positive blood culture for $A$. baumannii. The presence of acute vasculitis of chorionic fetal vessels is an indicator of fetal inflammatory response. No bacteria grew from the mother's urine sample. No inflammatory cells were seen in fetal lung parenchyma or GI tract lumen. Evidence of intrauterine fetal distress was seen, including grade II thymic involution and a moderate degree of erythroblastosis (nucleated red blood cells) in the 
54 Perinatal $A$. baumannii Infection He et al

Table 3 Summery of Postmortem Findings of Cases with Bacterial Cultures Positive for Acinetobacter Baumannii

\begin{tabular}{|l|l|l|l|l|l|}
\hline Case & $\begin{array}{l}\text { Gestational age } \\
\text { (wk) at birth }\end{array}$ & $\begin{array}{l}\text { Length of life } \\
\text { (postnatal age) }\end{array}$ & Cause of death & $\begin{array}{l}\text { Postmortem } \\
\text { bacterial culture }\end{array}$ & Placental findings \\
\hline 1 & 23 & $289 \mathrm{~d}$ & $\begin{array}{l}\text { Complications of } \\
\text { prematurity, } \\
\text { multiorgan failure }\end{array}$ & $\begin{array}{l}\text { Peritoneal } \\
\text { effusion }\end{array}$ & $\begin{array}{l}\text { Severe acute chorioamnionitis } \\
\text { with three-vessel acute } \\
\text { vasculitis and funisitis of } \\
\text { the umbilical cord and } \\
\text { acute vasculitis of chorionic } \\
\text { fetal blood vessels }\end{array}$ \\
\hline 2 & 21 & $<10 \mathrm{~min}$ & $\begin{array}{l}\text { Extreme prematurity and } \\
\text { amniotic fluid infection } \\
\text { syndrome }\end{array}$ & $\begin{array}{l}\text { Blood } \\
\text { and acute vasculitis of } \\
\text { chorionic fetal vessels }\end{array}$ \\
\hline 3 & 15 & 0 (stillbirth) & $\begin{array}{l}\text { Amniotic fluid infection } \\
\text { syndrome }\end{array}$ & $\begin{array}{l}\text { Blood and } \\
\text { lung }\end{array}$ & $\begin{array}{l}\text { Rod-shaped microorganisms } \\
\text { within umbilical blood vessel, } \\
\text { severe acute chorioamnionitis } \\
\text { with evidence of abruption }\end{array}$ \\
\hline
\end{tabular}

fetal blood vessels in both placenta and fetal organs. No dysmorphisms were identified. Cause of death was determined as extreme prematurity and amniotic fluid infection syndrome.

The third postmortem case was a female stillborn at 15 weeks' gestation to 34-year-old gravida four, para two mother who had a history of previous intrauterine fetal demise at 14 weeks' gestation. Postmortem toe-heel length was more consistent with 13 weeks' gestational age. Fetal weight was $25 \mathrm{~g}$, less than 3rd percentile (Hadlock Fetal Growth Curve).
Postmortem examination revealed a moderately macerated fetus with both blood and lung cultures positive for $A$. baumannii. Placenta showed rod-shaped microorganisms within the umbilical blood vessel (-Fig. 1C), along with severe acute chorioamnionitis with evidence of abruption. Gram stain of the umbilical cord vessel demonstrated the presence of gram-negative rods (-Fig. 1D). The mother's urine culture did not grow any bacteria. No dysmorphic features were seen. Cytogenetic study revealed a normal female karyotype.
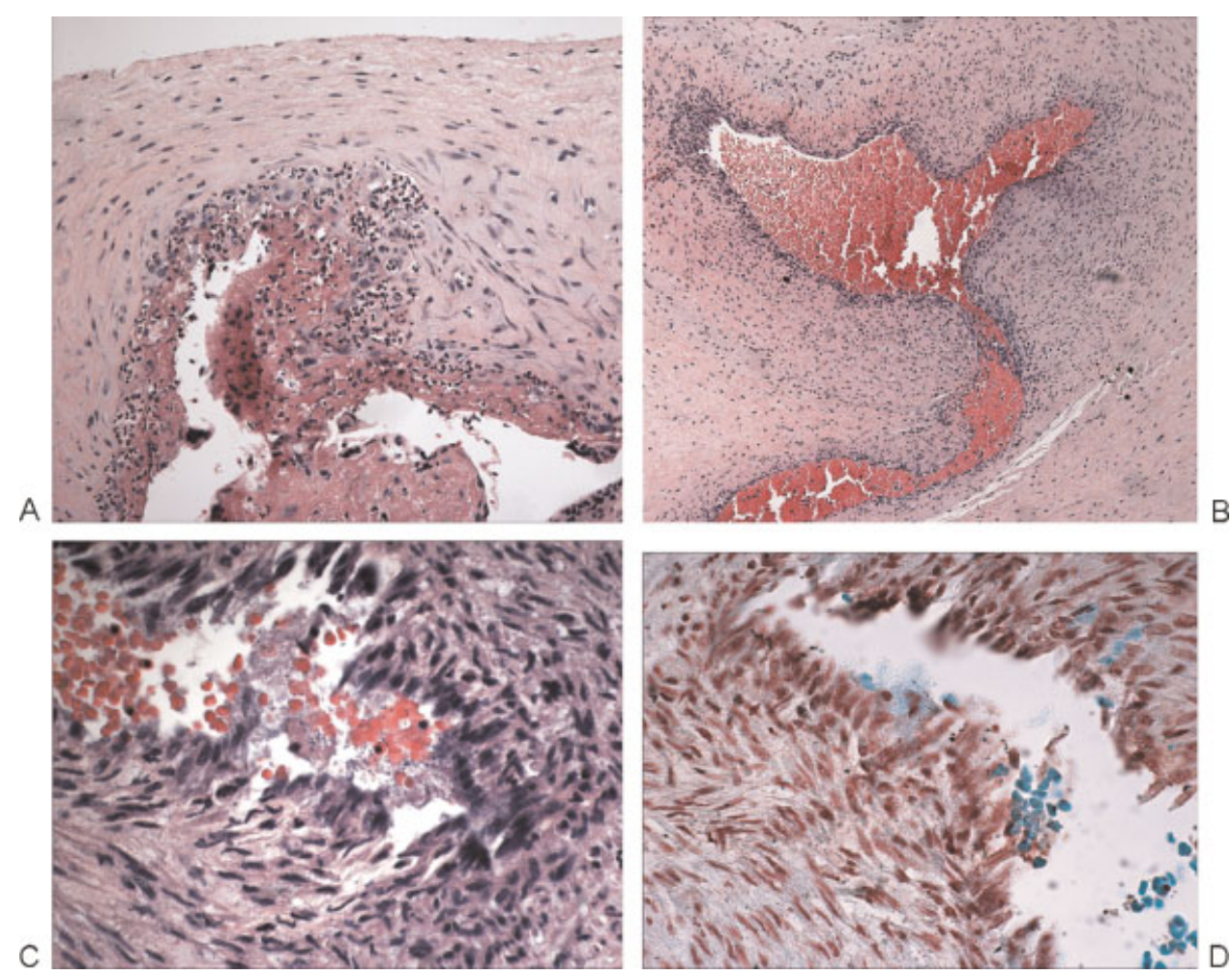

Fig. 1 Placental examinations in two perinatal autopsies (cases 2 and 3). (A) Acute chorioamnionitis at chorionic plate, case 2 (hematoxylin and eosin $[\mathrm{H \& E}], 100 \times$ ). (B) Acute vasculitis (inflammation of vascular wall) of chorionic fetal blood vessel, case $2(\mathrm{H} \& \mathrm{E}, 200 \times$ ). (C) Rod-shaped bacterial microorganisms in the lumen of umbilical cord blood vessel, case 3 (H\&E, $600 \times$ ). (D) Gram stain of (C), indicting gram-negative rods (Gram stain, $600 \times$ ). 


\section{Discussion}

The genus Acinetobacter comprises of many species (32 species at present). A. baumannii is the clinically most significant species because it is usually the most commonly isolated, especially from blood culture, and has the most severe clinical course. ${ }^{9,10}$ It is a gram-negative nonfermentative coccobacillus. A. baumannii has different shape depending on its stage of growth. During growth, the bacterium is rod-shaped and becomes rounder and shorter during its stationary phase. ${ }^{11}$

A wide spectrum of antibiotics resistance (so called "pan-resistant") was seen with A. baumannii. Contributing mechanisms include the low permeability of the outer membrane to antibiotics and certain efflux pumps. ${ }^{1,3}$ This pan-resistance led to increasing number of reports of associated morbidity and mortality worldwide, although some conflicting conclusions exist. One challenge of determining the clinical impact of positive $A$. baumannii culture results is to distinguish colonization (acquisition) versus infection. ${ }^{2,3}$ This study attempted to investigate the clinical outcomes with pathological evidence. Positive culture with histopathologic evidence of infection/inflammation suggests more likely significant infection, instead of acquisition.

Although during the recent decade A. baumannii has emerged as an important nosocomial pathogen, there is very limited number of studies in the United States reviewing the clinical outcome of $A$. baumannii infection in pregnancy. Aivazova et al reported a case of cervical A. baumannii infection during pregnancy that led to preterm cesarean section at 31 weeks' gestation. ${ }^{6}$ In this study, among the seven positive cultures during pregnancy, four cultures (from three patients) were sampled either around the time of labor (or procedure) or within 4 weeks before labor. Three cultures grew pure A. baumannii and one grew mixture with $E$. coli. Two cases had placental examination. In case, acute chorioamnionitis was seen; in case 6 , no evidence of infection/ inflammation was seen. This might be due to the antibiotic use in case 6. A. baumannii infection in these three cases was considered "most likely" relevant to the pregnancy outcomes (i.e., a significant contributing factor). Adverse outcomes were seen in all three cases with positive $A$. baumannii culture close to or around the time of labor.

The immature development of the immune state in premature neonates, the main population in the NICU, make them more vulnerable to $A$. baumannii infection. In particular, infants with very low birth weight are at high risk. McGrath et al described an outbreak in the NICU with six infants infected. ${ }^{4}$ All infants were premature. Postnatal age ranged from 10 to 197 days. One infant died of causes not attributed to infection of the organism. Simmonds et al reported a NICU outbreak with seven neonates infected by a same clone. ${ }^{5}$ This outbreak only infected $<750$-g neonates, and $\leq 26$ weeks' gestational age or $\leq 7$ days' postnatal age. Four of the seven infants died. No autopsy was reported.

This study identified three positive cultures from newborn units, including a wound culture from a full-term baby and two cultures from preterm NICU patients. All these neonates had a birth weight more than $750 \mathrm{~g}$. The three cases of perinatal death had a birth weight all below $750 \mathrm{~g}$. This comparison supported previous opinion that birth weight is a significant contributing factor to the survival of neonates with positive $A$. baumannii culture. ${ }^{5}$

In postmortem case 1 , the clinical significance of positive A. baumannii growing with other bacteria from peritoneal effusion cannot be determined. Most likely, this is a case of acquisition. In case 2, histological evidence of fetal inflammatory response was observed alongside the positive pure blood culture. In case 3, although there was no evidence of fetal inflammatory response, bacteria were seen in the lumen of fetal blood vessels of the umbilical cord. Furthermore, fetal inflammatory response is rarely seen in cases below 20 weeks' gestational age. In both case, there was no positive culture from the mother. Another issue mentioned previously is that $A$. baumannii has a different shape depending on its stage of growth. This may explain the rod shape observed in the lumen of umbilical cord blood vessels.

This report describes the experience from a single institute. The number of cases is limited. However, this is the first case series report of pregnancy-related and perinatal death cases with pathological study results. In all three pregnancies with positive A. baumannii culture close to or around the time of labor, one $\mathrm{SAB}$, one preterm labor, and one full-term with acute chorioamnionitis were seen. These results suggest a role of maternal A. baumannii infection in these adverse pregnancy outcomes. The stillborn or neonates were born following PPROM in the second trimester. All placentas showed severe chorioamnionitis. Pure cultures were found in two cases. Pure cultures were from both blood and fetal tissue. These results suggest a possible role of perinatal A. baumannii infection in PPROM and premature delivery resulting in fetal or neonatal demise.

\section{References}

1 Neonakis IK, Spandidos DA, Petinaki E. Confronting multidrugresistant Acinetobacter baumannii: a review. Int J Antimicrob Agents 2011;37:102-109

2 Falagas ME, Bliziotis IA, Siempos II. Attributable mortality of Acinetobacter baumannii infections in critically ill patients: a systematic review of matched cohort and case-control studies. Crit Care 2006;10:R48

3 Gordon NC, Wareham DW. Multidrug-resistant Acinetobacter baumannii: mechanisms of virulence and resistance. Int J Antimicrob Agents 2010;35:219-226

4 McGrath EJ, Chopra T, Abdel-Haq N, et al. An outbreak of carbapenem-resistant Acinetobacter baumannii infection in a neonatal intensive care unit: investigation and control. Infect Control Hosp Epidemiol 2011;32:34-41

5 Simmonds A, Munoz J, Aguero-Rosenfeld M, et al. Outbreak of Acinetobacter infection in extremely low birth weight neonates. Pediatr Infect Dis J 2009;28:210-214

6 Aivazova V, Kainer F, Friese K, Mylonas I. Acinetobacter baumannii infection during pregnancy and puerperium. Arch Gynecol Obstet 2010;281:171-174

7 Pinar H, Koch MA, Hawkins H, et al. The Stillbirth Collaborative Research Network Postmortem Examination Protocol. Am J Perinatol 2012;29:187-202 
56 Perinatal A. baumannii Infection He et al

8 Pinar H, Koch MA, Hawkins H, et al. The Stillbirth Collaborative Research Network (SCRN) placental and umbilical cord examination protocol. Am J Perinatol 2011;28:781-792

9 Wareham DW, Bean DC, Khanna P, et al. Bloodstream infection due to Acinetobacter spp: epidemiology, risk factors and impact of multi-drug resistance. Eur J Clin Microbiol Infect Dis 2008;27: 607-612
10 Wisplinghoff $\mathrm{H}$, Edmond MB, Pfaller MA, Jones RN, Wenzel RP, Seifert H. Nosocomial bloodstream infections caused by Acinetobacter species in United States hospitals: clinical features, molecular epidemiology, and antimicrobial susceptibility. Clin Infect Dis 2000;31:690-697

11 Nagata K, Mino H, Yoshida S. [Usefulness and limit of Gram staining smear examination]. Rinsho Byori 2010;58:490-497 (Japanese) 